\title{
EPEL promotes the migration and invasion of osteosarcoma cells by upregulating ROCK1
}

\author{
SHUNGUANG CHEN, ZHENGJIE LIU, SHENGJUN LU and BIAO HU \\ Department of Orthopaedics, Jingzhou Central Hospital, Jingzhou, Hubei 434020, P.R. China
}

Received April 24, 2018; Accepted December 18, 2018

DOI: $10.3892 /$ ol.2019.9975

\begin{abstract}
E2F-mediated cell proliferation enhancing long non-coding RNA (lncRNA) (EPEL) is a newly identified lncRNA involved in the regulation of lung cancer cell proliferation. However, its association with other types of cancer is unknown. The present study recruited patients with osteosarcoma and healthy controls. Tumor and adjacent healthy tissues were obtained from patients with osteosarcoma, and whole blood was extracted from patients and healthy controls. The expression levels of EPEL in tissues were detected by reverse transcription-quantitative polymerase chain reaction. The diagnostic value of serum EPEL for osteosarcoma was evaluated by receiver operating characteristic curve analysis. The association between serum levels of EPEL and basic clinical patient information was analyzed by $\chi^{2}$ test. Subsequently, EPEL overexpression in osteosarcoma cell lines was established, and its effects on cell migration and invasion were explored by Transwell assay. The implications of EPEL overexpression on Rho-associated coiled-coil containing protein kinase 1 (ROCK1) expression were investigated by western blotting. The results revealed that EPEL was upregulated in tumor tissues compared with adjacent tissues. In addition, serum levels of EPEL were higher in patients with osteosarcoma compared with healthy controls, and were positively associated with distant tumor metastasis. Furthermore, EPEL overexpression promoted the migration and invasion of osteosarcoma cells and induced overexpression of ROCK1. In conclusion, these results suggested that EPEL may promote the migration and invasion of osteosarcoma cells by upregulating ROCK1.
\end{abstract}

Correspondence to: Dr Shunguang Chen, Department of Orthopaedics, Jingzhou Central Hospital, 60 Jingzhong Road, Jingzhou, Hubei 434020, P.R. China

E-mail: rbnzs97@163.com

Key words: osteosarcoma, E2F-mediated cell proliferation enhancing long non-coding RNA, migration, Rho-associated coiled-coil containing protein kinase 1 , invasion

\section{Introduction}

Osteosarcoma, also known as osteogenic sarcoma, is the most common type of bone malignancy. Despite its low incidence in the general population $(<1 / 100,000)$, osteosarcoma is one of the primary causes of cancer-associated mortality among young adults and children $(1,2)$. Osteosarcoma mainly affects long bones, and commonly develops in the distal femur (43\%), proximal tibia (23\%) and humerus (10\%) (3). Patients with osteosarcoma usually suffer from swelling combined with severe pain in the affected bone (4). Due to great efforts being made regarding the prevention and treatment of this disease, the long-term survival rate of patients has increased to $70 \%$; however, $\sim 20 \%$ of patients exhibit distant tumor metastasis at the time of diagnosis, which markedly impairs their survival (4). The early diagnosis of osteosarcoma and the development of efficient treatment are therefore critical to improve the survival rate of patients with osteosarcoma.

Osteosarcoma progression is a complex and multi-step process involving numerous external and internal factors (5). It has been revealed that progression of osteosarcoma is accompanied by alterations in the expression pattern of a large set of long non-coding RNAs (lncRNAs) (6). IncRNAs consist of $>200$ nucleotides and have critical functions in normal and pathological processes (7-9). E2F-mediated cell proliferation enhancing lncRNA (EPEL) is a novel lncRNA involved in the regulation of lung cancer cell proliferation (10); however, its role in other types of cancer remains unknown. In the present study, the role of EPEL in osteosarcoma was investigated. The results demonstrated that EPEL may promote the migration and invasion of osteosarcoma cells by upregulating Rho-associated coiled-coil containing protein kinase 1 (ROCK1).

\section{Materials and methods}

Patients. The present study recruited 39 patients with osteosarcoma who were diagnosed and treated at the Jingzhou Central Hospital (Hubei, China) between March 2009 and January 2013. Following diagnosis, patients with other types of malignancies or severe diseases were not included. The 39 patients included 22 men and 17 women, aged between 11 and 54 years (mean age, 32 \pm 7.7 years). Distant tumor metastases were found in 22 cases. In addition, 42 healthy volunteers were included as the control group. This group included 22 men and 20 women, aged between 15 and 52 years (mean 


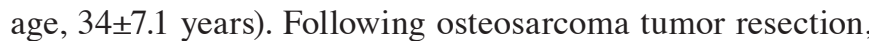
all patients were followed-up for 5 years to record survival conditions. The study was approved by the Ethics Committee of the Jingzhou Central Hospital, and all patients and their guardians provided written informed consent.

Specimen collection. Patients with osteosarcoma underwent surgical tumor resection. Tumor tissues and adjacent healthy tissues ( $5 \mathrm{~cm}$ around the tumor) were collected during surgical resection. Whole blood $(10 \mathrm{ml})$ was extracted from the elbow vein of patients and healthy controls. Blood was maintained at room temperature for $2 \mathrm{~h}$, and centrifuged at $1,175 \mathrm{x} \mathrm{g}$ for $20 \mathrm{~min}$ to collect serum. All specimens were stored in liquid nitrogen for long-term use.

Cell lines and cell culture. The normal bone cell line hFOB, and osteosarcoma cell lines U2OS, MG-63 and SAOS-2, were purchased from the American Type Culture Collection (ATCC, Manassas, VA, USA). The SAOS-2 and MG-63 cell lines were cultured in Eagle's minimum essential medium (cat. no. 30-2003; ATCC) supplemented with 10\% heat-inactivated fetal bovine serum (FBS; Sigma-Aldrich; Merck KGaA, Darmstadt, Germany). The U2OS and hFOB cell lines were cultured in ATCC-formulated McCoy's 5a medium (cat. no. 30-2007; ATCC) supplememted with $10 \%$ FBS. All cells were cultured at $37^{\circ} \mathrm{C}$ in a humidified incubator containing $5 \% \mathrm{CO}_{2}$. No serum was added to the culture media during treatment with Stemolecule ${ }^{\mathrm{TM}}$ ROCK I Inhibitor (10 nM; cat. no. 203911-26-6; Stemgent, Inc., Cambridge, MA, USA).

Reverse transcription-quantitative polymerase chain reaction $(R T-q P C R)$. Tumor and adjacent healthy tissues were ground in liquid nitrogen, and incubated with TRIzol ${ }^{\circledR}$ reagent (Invitrogen; Thermo Fisher Scientific, Inc., Waltham, MA, USA), in order to extract total RNA. TRIzol ${ }^{\circledR}$ reagent was directly mixed with serum samples and in vitro cultivated cells to extract total RNA. The NanoDrop ${ }^{\text {TM }} 2000$ Spectrophotometer (NanoDrop; Thermo Fisher Scientific, Inc., Wilmington, DE, USA) was used to determine the quantity and quality of extracted RNA. The RNA samples of satisfactory quality (A260/A280 between 1.8 and 2.0) were subjected to reverse transcription using SuperScript III Reverse Transcriptase (Thermo Fisher Scientific, Inc.) to synthesize cDNA according to the manufacturer's protocol. The PCR reaction system was prepared using $\mathrm{SYBR}^{\circledR}$-Green Real-Time PCR Master Mixes (Thermo Fisher Scientific, Inc.) with the following primers: EPEL forward, 5'-GAGGCAGACCAC GTGAGAG-3' and reverse, 5'-CAGATTTAAACCCCGCAC TG-3'; $\beta$-actin forward, 5'-GACCTCTATGCCAACACAGT-3' and reverse, 5'-AGTACTTGCGCTCAGGAGGA-3'. PCR reactions were conducted using a CFX96 Touch ${ }^{\mathrm{TM}}$ Real-Time PCR Detection system (Bio-Rad Laboratories, Inc., Hercules, CA, USA) with the following reaction conditions: $95^{\circ} \mathrm{C}$ for $50 \mathrm{sec}$, followed by 40 cycles at $95^{\circ} \mathrm{C}$ for $10 \mathrm{sec}$ and $60^{\circ} \mathrm{C}$ for $40 \mathrm{sec}$. Data analysis was performed using the $2^{-\Delta \Delta \mathrm{Cq}}$ method (11) and EPEL expression was normalized to the endogenous control $\beta$-actin.

Construction of EPEL expression vector and transfection. Full length EPEL cDNA was provided by Sangon Biotech
Co., Ltd., (Shanghai, China) and inserted into a pIRSE2-EGFP vector (Clontech Laboratories, Inc., Mountainview, CA, USA) to construct an EPEL expression vector. The EPEL small interfering (si)RNA, 5'-UACAAAACUCUGGAACCU C(dTdT)-3' and negative control siRNA, 5'-CCUACGCCA CCAAUUUCGU(dTdT)-3' were synthesized by Shanghai GenePharma Co., Ltd. (Shanghai, China). U2OS, MG-63 and SAOS-2 cells were cultured overnight to reach $80-90 \%$ confluence prior to transfection. Lipofectamine ${ }^{\circledR} 2000$ reagent (cat. no. 11668-019; Invitrogen; Thermo Fisher Scientific, Inc.) was used to transfect cells $\left(5 \times 10^{5} /\right.$ sample) with $10 \mathrm{nM}$ vector or $50 \mathrm{nM}$ siRNA. Transfection with an empty vector or negative control siRNA was used as a negative control. Overexpression rate $>200 \%$ and knockdown rate $<50 \%$ were confirmed by RT-qPCR compared with control cells.

Cell migration and invasion assays. Cells were collected during the logarithmic growth phase $24 \mathrm{~h}$ post-transfection, and single cell suspensions of $5 \times 10^{4}$ cells $/ \mathrm{ml}$ were prepared. Cell migration and invasion were measured by Transwell migration and invasion assays. For the migration assay, $5 \times 10^{4}$ cells in $0.1 \mathrm{ml}$ serum-free culture medium were added into the upper chamber, and the lower chamber was filled with RPMI-1640 medium (Gibco; Thermo Fisher Scientific, Inc.) supplemented with $20 \%$ fetal calf serum (Sigma-Aldrich; Merck KGaA). After $24 \mathrm{~h}$, membranes were collected and stained with $0.5 \%$ crystal violet (Sigma-Aldrich; Merck KGaA) at room temperature for $20 \mathrm{~min}$. The same procedure was followed for the invasion assay, with the exception that the upper chamber was pre-coated with Matrigel (cat. no. 356234; EMD Millipore, Billerica, MA, USA). Cells were observed using the CX33 optical microscope (Olympus Corporation, Tokyo, Japan). In cases of Stemolecule ${ }^{\mathrm{TM}}$ ROCK I Inhibitor (10 nM; cat. no. 203911-26-6; Stemgent, Inc.) treatment, cells were pretreated with Stemolecule ${ }^{\mathrm{TM}}$ ROCK I Inhibitor for $12 \mathrm{~h}$ at $37^{\circ} \mathrm{C}$ in a humidified incubator containing $5 \% \mathrm{CO}_{2}$ before use.

Western blotting. Cells were collected 3 days post-transfection. Cells were mixed with radioimmunoprecipitation assay lysis and extraction Buffer (Thermo Fisher Scientific, Inc.) on ice to extract the total protein. The bicinchoninic acid method was used to quantify protein concentration. SDS-PAGE was performed with a $10 \%$ gel (20 $\mu$ g protein loaded per lane), followed by protein transfer onto polyvinylidene fluoride membranes. Membranes were blocked with 5\% skimmed milk for $1 \mathrm{~h}$ at room temperature, followed by incubation with rabbit anti-ROCK1 (cat. no. ab45171; 1:2,000; Abcam, Cambridge, UK) and anti- $\beta$-actin (cat. no. ab8227; 1:1,000; Abcam) primary antibodies overnight at $4^{\circ} \mathrm{C}$. Membranes were subsequently incubated with the anti-rabbit immunoglobulin G-horseradish peroxidase secondary antibody (cat. no. MBS435036; 1:1,000; MyBioSource, San Diego, CA, USA) or goat anti-mouse IgG (H+L, cat. no. A-11001, Thermo Fisher Scientific, Inc.) for $2 \mathrm{~h}$ at room temperature. Enhanced chemiluminescence detection reagent (Sigma-Aldrich; Merck KGaA) was used to measure signal development. Relative expression levels of ROCK1 were normalized to the endogenous control $\beta$-actin using ImageJ v1.46 (National Institutes of Health, Bethesda, MD, USA). 


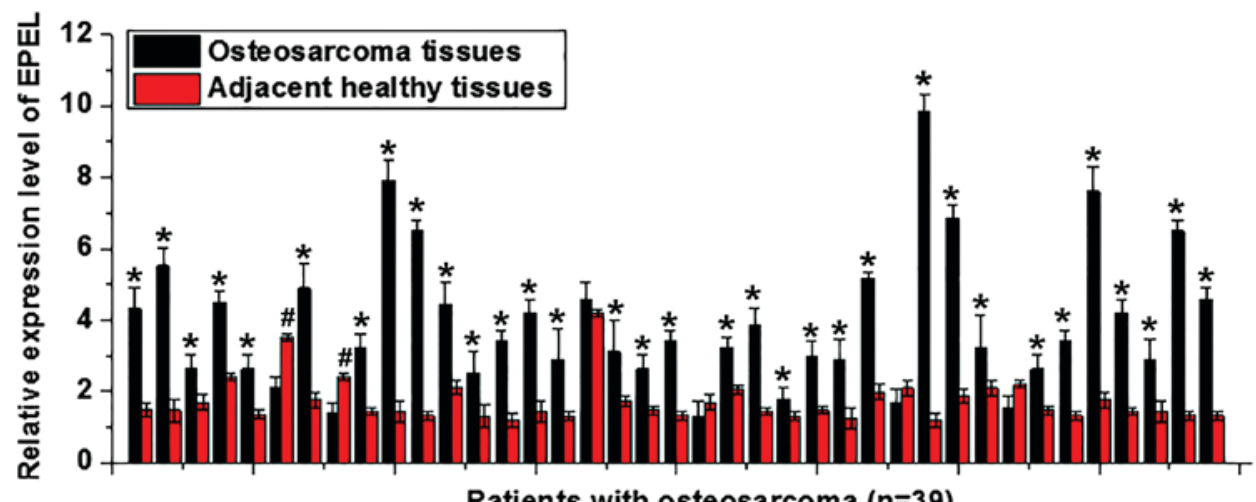

Patients with osteosarcoma $(n=39)$

Figure 1. Expression levels of EPEL in osteosarcoma tissues and adjacent healthy tissues collected from 39 patients. ${ }^{*} \mathrm{P}<0.05$ vs. adjacent healthy tissue from the same patient; " $\mathrm{P}<0.05$ vs. osteosarcoma tissue collected from the same patient. EPEL, E2F-mediated cell proliferation enhancing long non-coding RNA.

Statistical analysis. SPSS 19.0 (IBM Corp., Armonk, NY, USA) was used for all statistical analyses. All data are presented as the means \pm standard deviation, and comparisons among multiple groups were performed using one-way analysis of variance and the least significant difference test. The receiver operating characteristic (ROC) curve analysis was performed to evaluate the diagnostic value of serum EPEL for osteosarcoma. Patients were then divided into the high expression group $(n=20)$ and low expression group $(n=19)$ according to the median serum levels of EPEL. Survival curves of these two groups were plotted using the Kaplan-Meier method, and compared by log rank test. Categorical data were processed by $\chi^{2}$ test. $\mathrm{P}<0.05$ was considered to indicate a statistically significant difference.

\section{Results}

Expression of EPEL in tumor and adjacent healthy tissues of 39 patients with osteosarcoma. EPEL expression levels in osteosarcoma and adjacent healthy tissues were detected by RT-qPCR. As illustrated in Fig. 1, in samples from $~ 85 \%$ (33/39) of patients, the expression levels of EPEL were upregulated in tumor tissues compared with adjacent healthy tissues $(\mathrm{P}<0.05)$. A total of 2 patients had significantly higher expression levels of EPEL in adjacent healthy tissues compared with those in tumor tissues $(\mathrm{P}<0.05)$. No significant differences were observed in the remaining three patients $(\mathrm{P}>0.05)$. The upregulation of EPEL may therefore be involved in the pathogenesis of osteosarcoma.

Expression levels of EPEL in the serum of healthy controls and patients with osteosarcoma, and diagnostic and prognostic values. Distant tumor metastasis was observed in 22 patients, who formed the distant metastasis (DM) group. The remaining 17 patients formed the non-distant metastasis (non-DM) group. The expression levels of EPEL in the two groups were detected by RT-qPCR. As illustrated in Fig. 2A, serum levels of EPEL were higher in the DM and non-DM groups compared with the control group $(\mathrm{P}<0.05)$. In addition, serum levels of EPEL were higher in the DM group compared with the non-DM group $(\mathrm{P}<0.05)$. The receiver operating characteristic $(\mathrm{ROC})$ curve analysis was performed to evaluate the diagnostic value of serum EPEL for osteosarcoma. As shown in Fig. 2B, the area under the curve was 0.8817 , with a $95 \%$ confidence interval of 0.8111-0.9523 $(\mathrm{P}<0.0001)$, suggesting that serum EPEL may serve as a potential biomarker for osteosarcoma. Patients were then divided into the high expression $(\mathrm{n}=20)$ and low expression $(n=19)$ groups according to the median serum levels of EPEL. Survival curves of these two groups were plotted using the Kaplan-Meier method and compared by log rank test. As illustrated in Fig. 2C, the overall survival rate of patients in the high expression group was significantly lower than that in the low expression group $(\mathrm{P}=0.015)$, suggesting that high serum levels of EPEL may be associated with poor survival of patients with osteosarcoma.

Association between serum levels of EPEL and clinical data of patients with osteosarcoma. Associations between serum levels of EPEL (high and low) and the clinical data of patients with osteosarcoma were analyzed by $\chi^{2}$ test. As displayed in Table I, serum levels of EPEL were not associated with sex, age, tumor size or lifestyle habits, including smoking and drinking. However, EPEL serum levels were significantly associated with distant tumor metastasis.

EPEL overexpression upregulates the expression of ROCKI in osteosarcoma cell lines, but not in a normal bone cell line. Based on the aforementioned data, it was hypothesized that EPEL may be involved in osteosarcoma metastasis. ROCK1 is known to be involved in the migration and invasion of osteosarcoma cells (10). In the present study, the EPEL expression vector and siRNA were transfected into all cell lines prior to assessing ROCK1 expression levels. As displayed in Fig. 3, EPEL overexpression (Fig. 3A) and silencing (Fig. 3B) were reached following transfection in all cell lines. EPEL overexpression significantly upregulated the expression of ROCK1 in U2OS, MG-63 and SAOS-2 cell lines $(\mathrm{P}<0.05)$, but not in the hFOB cell line (Fig. 3C). In addition, EPEL siRNA-induced silencing significantly downregulated the expression of ROCK1 in U2OS, MG-63 and SAOS-2 cell lines $(\mathrm{P}<0.05)$, but not in hFOB cells (Fig. 3D).

EPEL overexpression promotes migration and invasion of osteosarcoma cells possibly by upregulating ROCK1. Transwell migration and invasion assays were performed to investigate the effects of EPEL overexpression on cell migration 
Table I. Association between serum levels of EPEL and clinical data of patients with osteosarcoma.

\begin{tabular}{|c|c|c|c|c|c|}
\hline Variable & Cases & High-expression & Low-expression & $\chi^{2}$ & P-value \\
\hline Sex & & & & 0.69 & 0.41 \\
\hline Male & 22 & 10 & 12 & & \\
\hline Female & 17 & 10 & 7 & & \\
\hline Age (years) & & & & 0.65 & 0.42 \\
\hline$>35$ & 19 & 11 & 8 & & \\
\hline$<35$ & 20 & 9 & 11 & & \\
\hline Drinking & & & & 0.64 & 0.42 \\
\hline Yes & 27 & 15 & 12 & & \\
\hline No & 12 & 5 & 7 & & \\
\hline Smoking & & & & 0.63 & 0.43 \\
\hline Yes & 21 & 12 & 9 & & \\
\hline No & 18 & 8 & 10 & & \\
\hline Tumor diameter & & & & 1.34 & 0.24 \\
\hline$\geq 5 \mathrm{~cm}$ & 16 & 10 & 6 & & \\
\hline$<5 \mathrm{~cm}$ & 23 & 10 & 13 & & \\
\hline Distant metastasis & & & & 7.69 & 0.006 \\
\hline Yes & 22 & 15 & 5 & & \\
\hline No & 17 & 5 & 12 & & \\
\hline
\end{tabular}

EPEL, E2F-mediated cell proliferation enhancing long non-coding RNA.
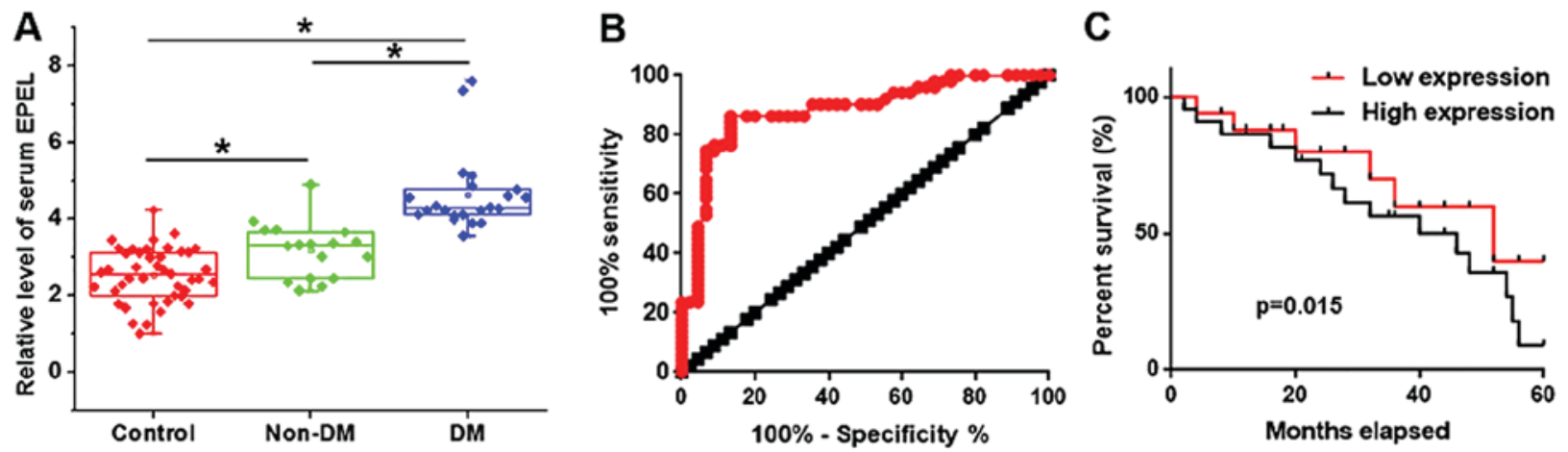

Figure 2. Expression of EPEL in serum samples from healthy controls and patients with osteosarcoma, and diagnostic and prognostic values. (A) Expression levels of EPEL in the serum of healthy controls and patients. (B) Diagnostic values of serum levels of EPEL in samples from patients with osteosarcoma analyzed by receiving operating characteristic curve analysis. (C) Comparison of survival curves of patients with high and low serum levels of EPEL. ${ }^{\mathrm{P}}<0.05$. DM, distant metastases; EPEL, E2F-mediated cell proliferation enhancing long non-coding RNA.

and invasion of the osteosarcoma cell lines U2OS, MG-63 and SAOS-2, and the normal bone cell line hFOB. As illustrated in Fig. 4, EPEL expression vector transfection significantly promoted cell migration and invasion of the osteosarcoma cell lines U2OS (Fig. 4A), MG-63 (Fig. 4B) and SAOS-2 (Fig. 4C) $(\mathrm{P}<0.05)$, but not the normal bone cell line hFOB (Fig. 4D) $(\mathrm{P}>0.05)$. However, cell treatment with Stemolecule ${ }^{\mathrm{TM}}$ ROCK I Inhibitor (10 nM; cat no. 203911-26-6; Stemgent, Inc.) significantly reduced the enhancing effects of EPEL overexpression on cell migration and invasion of osteosarcoma cell lines. Fig. 4E and F represent cell migration and invasion results corresponding to Fig. 4A, respectively. In addition, EPEL siRNA transfection significantly inhibited cell migration and invasion of osteosarcoma cell lines U2OS (Fig. 5A),
MG-63 (Fig. 5B) and SAOS-2 (Fig. 5C) $(\mathrm{P}<0.05)$, but not normal bone cell line hFOB (Fig. 5D) (P>0.05). Fig. 5E and $\mathrm{F}$ represent cell migration and invasion results corresponding to Fig. 5A, respectively. These data suggested that EPEL may promote cell migration and invasion of osteosarcoma cells by upregulating ROCK1.

\section{Discussion}

A previous study reported that EPEL is upregulated in lung cancer (10). The present study demonstrated that the expression levels of EPEL were significantly upregulated in the tissue samples of most patients with osteosarcoma. In addition, serum circulating levels of EPEL were significnatly 

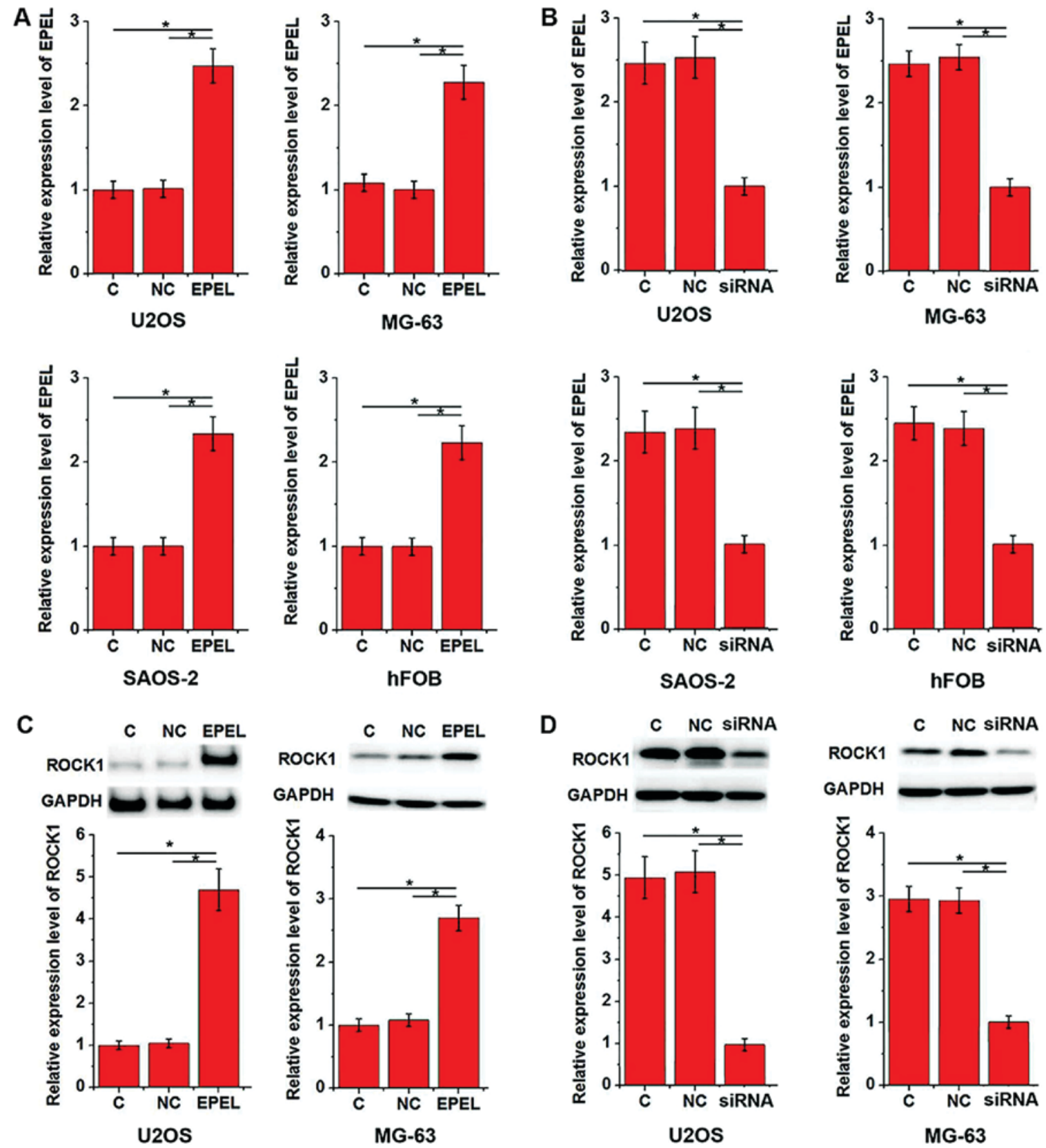

D
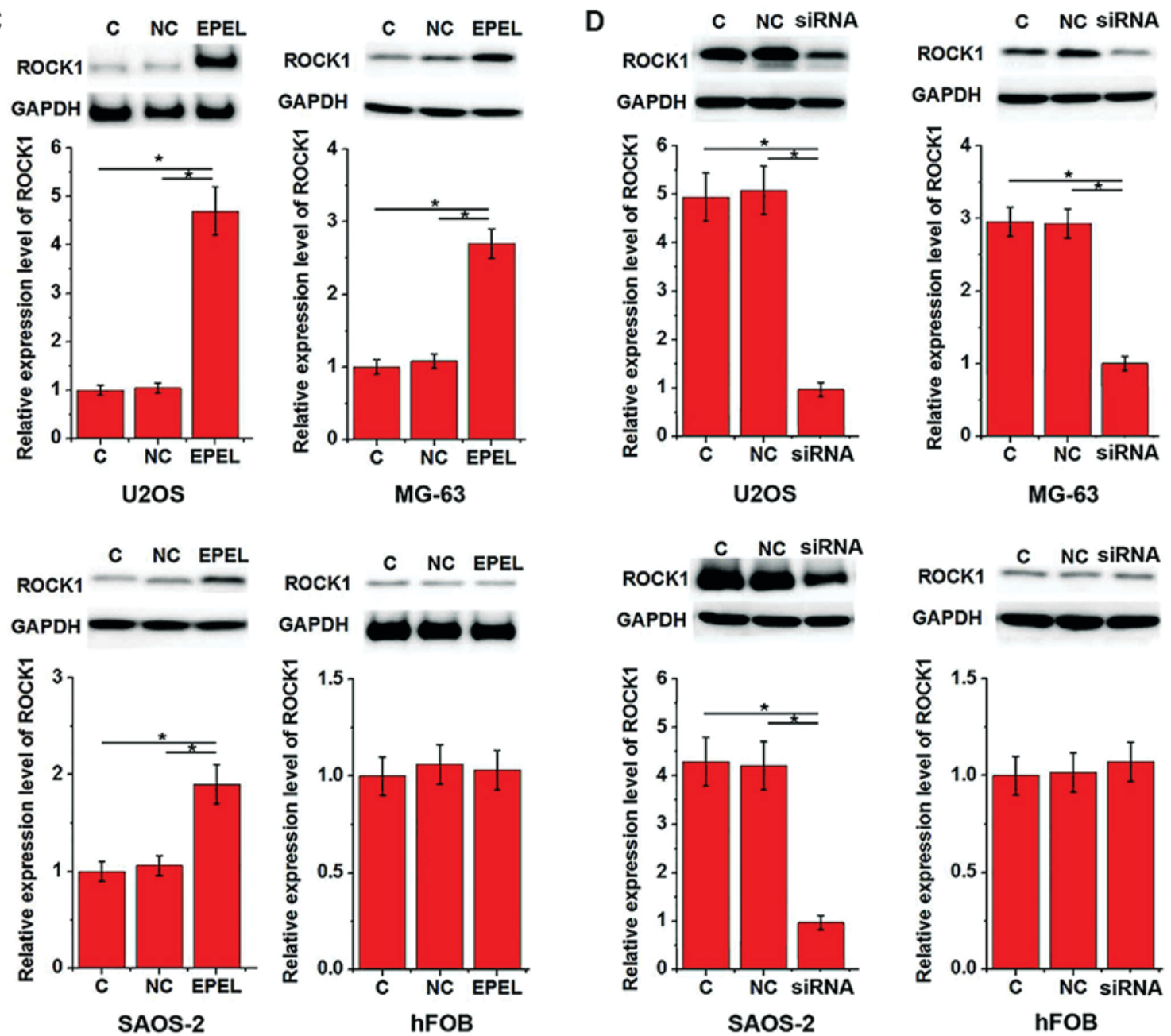

Figure 3. EPEL overexpression upregulates the expression of ROCK1 in osteosarcoma cell lines, but not in a normal bone cell line. Effects of (A) EPEL expression vector and (B) siRNA transfection on EPEL expression. Effects of EPEL (C) overexpression and (D) siRNA-induced silencing on ROCK1 expression in U2OS, MG-63 and SAOS-2 osteosarcoma cell lines, and hFOB normal bone cells. ${ }^{*} \mathrm{P}<0.05$. EPEL, E2F-mediated cell proliferation enhancing long non-coding RNA; C, control; NC, negative control; ROCK1, Rho-associated coiled-coil containing protein kinase 1; siRNA, silencing RNA. 
A
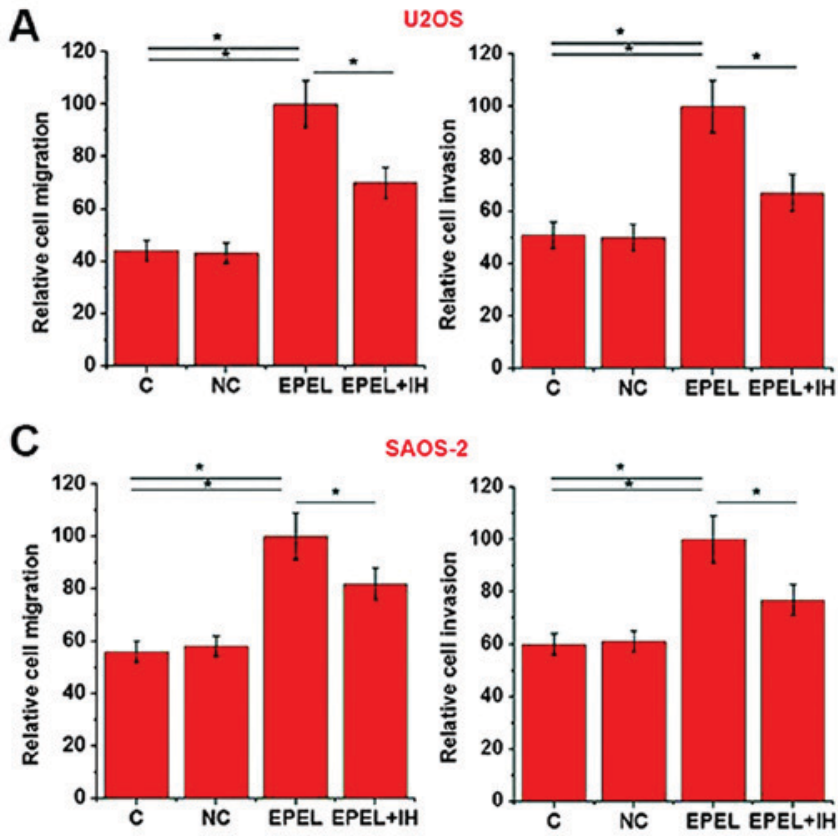

SAOS-2
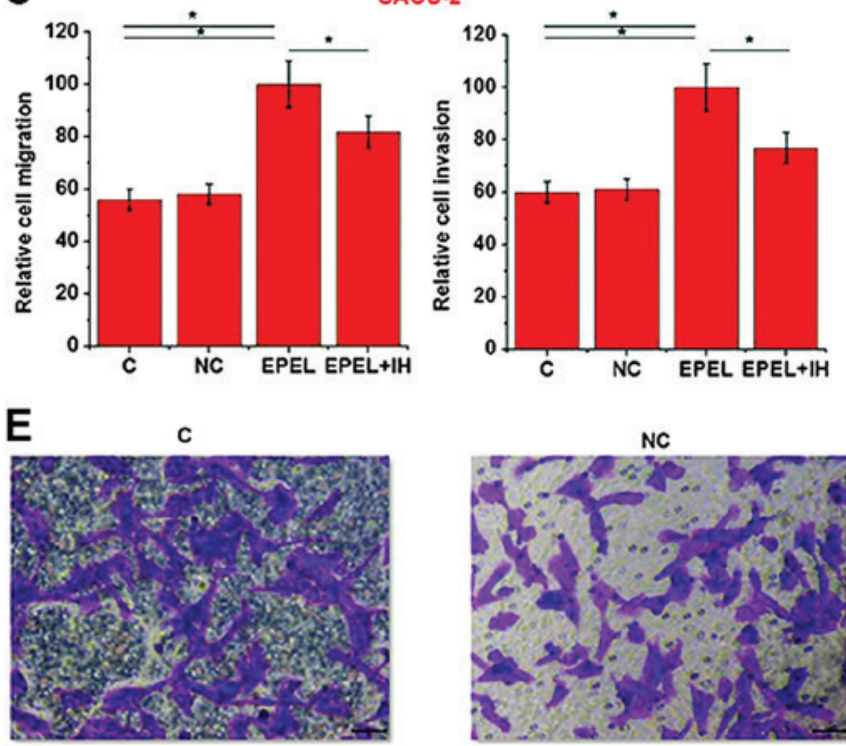

$\mathrm{F}$

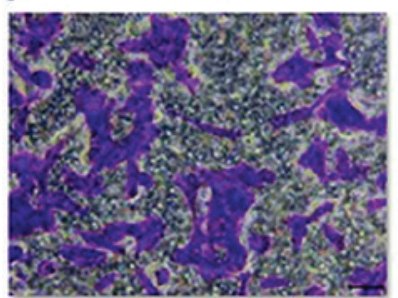

NC

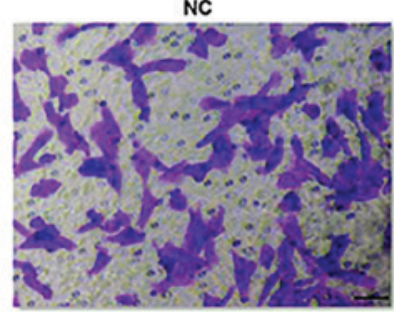

NC

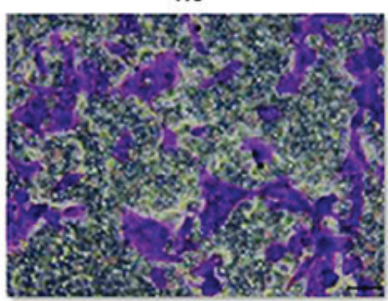

B

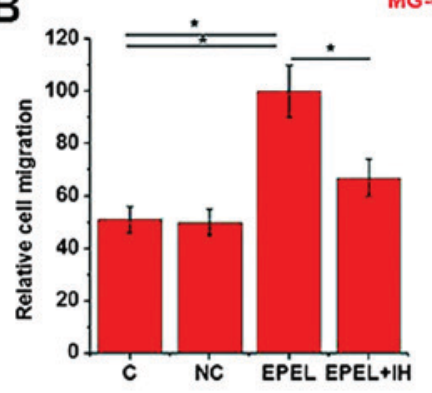

MG-63

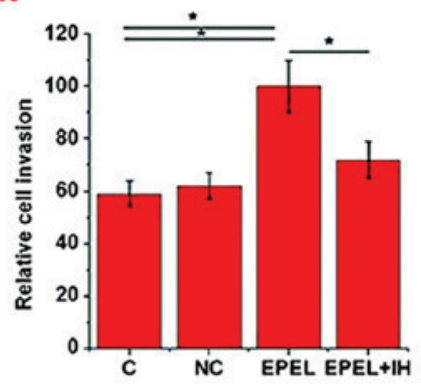

hFOB
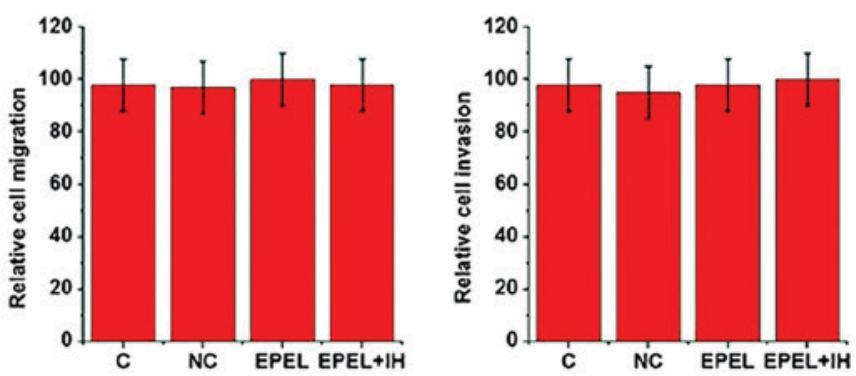

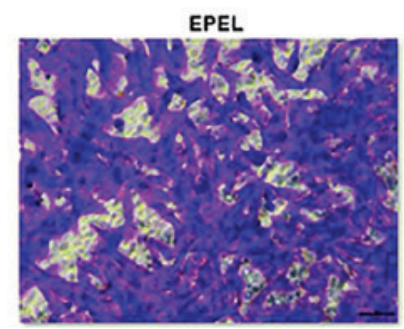

EPEL

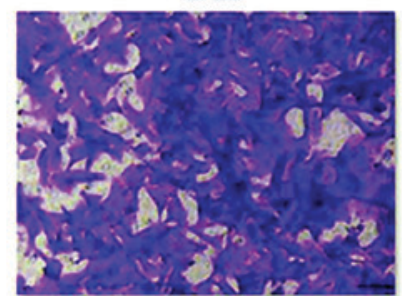

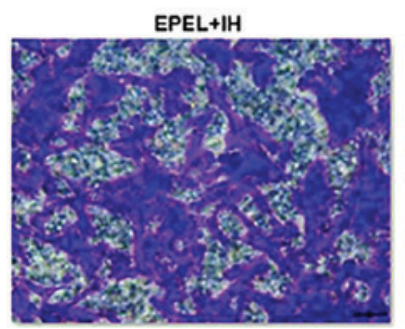

EPEL+IH

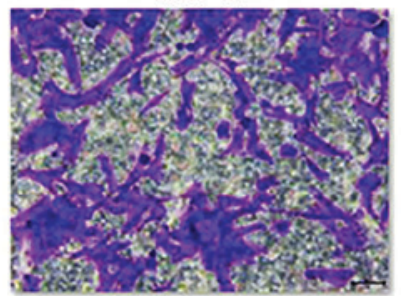

Figure 4. EPEL overexpression promotes migration and invasion of osteosarcoma cells, possibly by upregulating ROCK1. Effects of EPEL expression vector transfection on cell migration and invasion of osteosarcoma cell lines (A) U2OS, (B) MG-63 and (C) SAOS-2 and (D) normal bone cell line hFOB. (E) and (F) represent the representative cell migration and invasion data of (A) (magnification, $\mathrm{x} 40$ ), respectively. ${ }^{*} \mathrm{P}<0.05$. C, control; EPEL, E2F-mediated cell proliferation enhancing long non-coding RNA; IH, Stemolecule ${ }^{\mathrm{TM}}$ ROCKI Inhibitor; NC, negative control; ROCK1, Rho-associated coiled-coil containing protein kinase 1.

higher in patients with osteosarcoma than in healthy controls, suggesting that EPEL may serve as an oncogene in the development of osteosarcoma. In addition, a previous study has demonstrated that EPEL promotes lung cancer cell proliferation (10), indicating the stimulating effects of EPEL on tumor growth. In the present study, serum levels of EPEL were not significantly associated with tumor size, suggesting that EPEL may not be associated with osteosarcoma growth. Conversely, EPEL serum levels were significantly associated with distant tumor metastasis. In addition, transfection with an EPEL expression vector significantly promoted cell migration and invasion of osteosarcoma cell lines. These data indicated that EPEL may be involved in the regulation of osteosarcoma metastasis but not tumor growth. This suggested that the pathogenesis of osteosarcoma may be different from that of lung cancer.
According to previous studies, $20 \%$ of patients with osteosarcoma present distant tumor metastasis at the time of diagnosis; these patients have a poor prognosis $(4,12)$. Early diagnosis and treatment are therefore crucial for the survival of these patients. Development of human diseases is usually accompanied by blood marker modifications, and monitoring the changes in these markers aids in the diagnosis of human disease (13). In the present study, ROC curve analysis revealed that serum EPEL may be used to distinguish patients with osteosarcoma from healthy conrols. In addition, survial analysis indicated that high expression levels of EPEL were associated with poor patient survival. These data suggested that serum EPEL may serve as a potential diagnostic and prognostic biomarker for osteosarcoma. The expression of some lncRNAs however are affected by internal and external factors, including aging (14), alcohol consumption (15) 
A
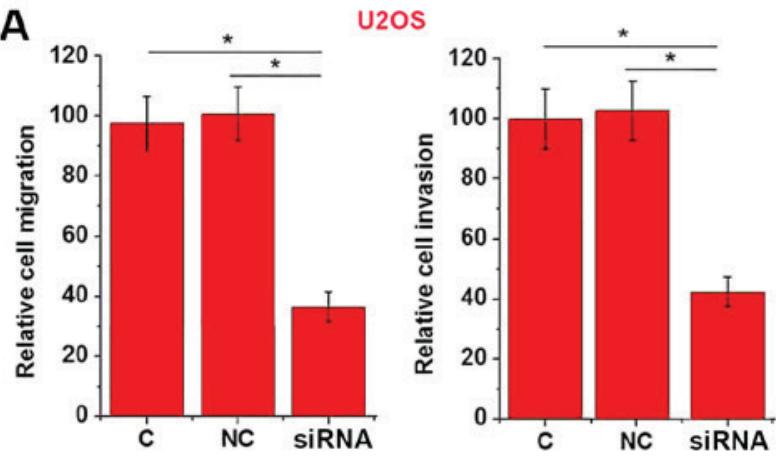

C

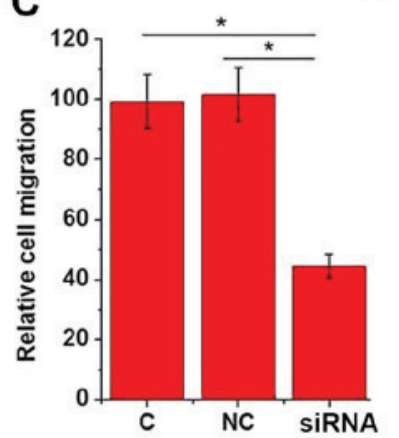

SAOS-2

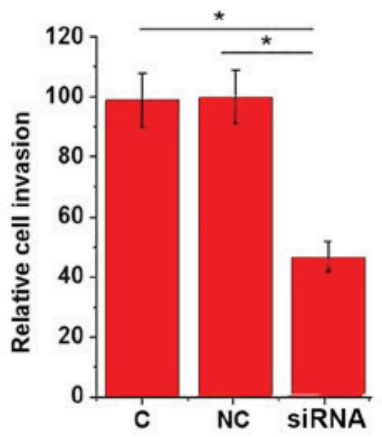

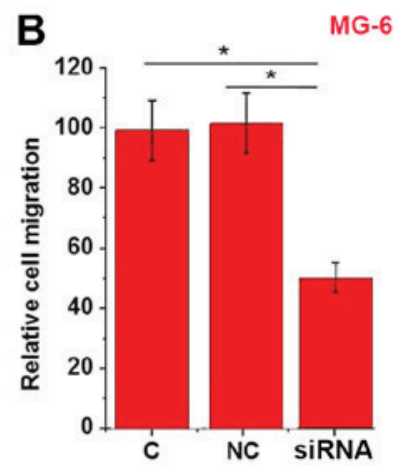

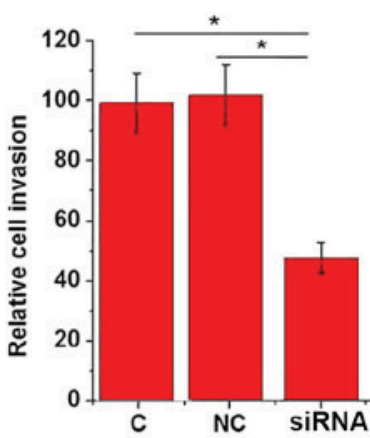

D

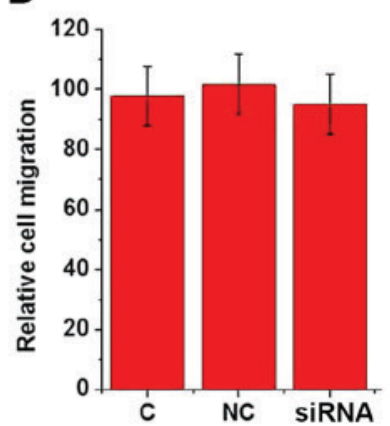

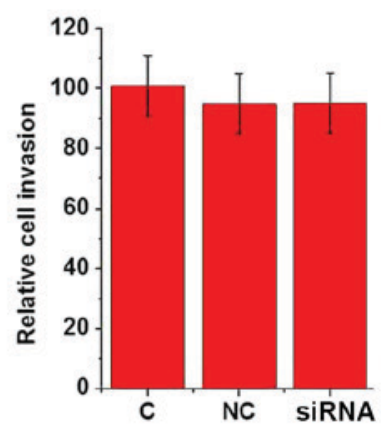

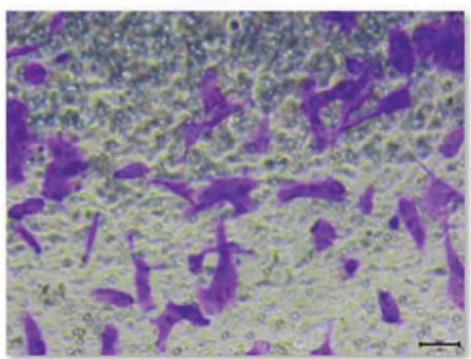

SiRNA

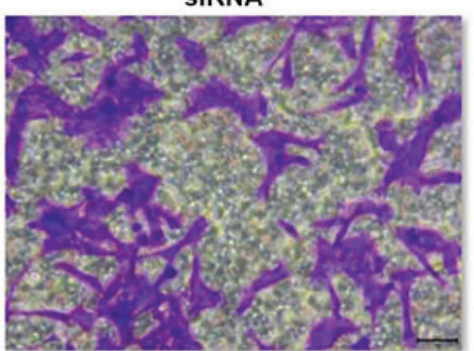

Figure 5. EPEL siRNA silencing inhibits the migration and invasion of osteosarcoma cells. The effects of EPEL siRNA transfection on cell migration and invasion of osteosarcoma cell lines (A) U2OS, (B) MG-63 and (C) SAOS-2, and (D) normal bone cell line hFOB. (E) and (F) represent the representative cell migration and invasion data of (A), respectively (magnification, $\mathrm{x} 40$ ). ${ }^{*} \mathrm{P}<0.05$. C, control; EPEL, E2F-mediated cell proliferation enhancing long non-coding RNA; NC, negative control; siRNA, silencing RNA.

and smoking (16), which can affect the accuracy of certain lncRNAs in the diagnosis of human diseases. In the present study, serum levels of EPEL were not associated with sex, age, tumor size or lifestyle habits, including smoking and drinking. This indicated that EPEL may be highly accurate in the diagnosis and prognosis of osteosarcoma. In addition, since EPEL is a novel lncRNA unkown in other diseases except lung cancer, the combined use of multiple biomarkers, such as alkaline phosphatase, may imporve the diagnosis and prognosis of osteosarcoma.
ROCK1 is a protein serine/threonine kinase with prominent functions in cancer cell motility, metastasis and angiogenesis (17). It is well known that ROCK1 is involved in the migration and invasion of osteosarcoma cells; inhibition of ROCK1 may therefore serve as a potential therapeutic target in the treatment of osteosarcoma $(10,18-20)$. In the present study, EPEL transfection significantly promoted the expression of ROCK1 in three osteosarcoma cell lines. In addition, cell treatment with a ROCK1 inhibitor significantly reduced the enhancing effects of EPEL overexpression on cell 
migration and invasion. These data suggested that EPEL may promote cell migration and invasion of osteosarcoma cells by upregulating ROCK1. Notably, EPEL overexpression had no effects on hFOB cells, suggesting that EPEL may serve a potential therapeutic target in the treatment of osteosarcoma. However, the study only elucidated EPEL-ROCK1 sequential signaling in osteosarcoma; whether this interaction is direct or indirect is still unknown and requires further investigation.

In conclusion, the present study revealed that EPEL was upregulated in osteosarcoma, and that serum levels of EPEL may serve as a promising diagnostic and prognostic marker for osteosarcoma. In addition, EPEL overexpression promoted the migration and invasion of osteosarcoma cells and ROCK1 expression, whereas siRNA silencing inhibited these phenomena. Conversely, cell treatment with a ROCK1 inhibitor reduced the enhancing effects of EPEL overexpression on cancer cell migration and invasion. These results suggested that EPEL may promote the migration and invasion of osteosarcoma cells by upregulating ROCK1. Due to the low incidence of this disease, only 39 patients were included in this study. Future studies with a larger sample size are required to confirm the present findings.

\section{Acknowledgements}

Not applicable.

\section{Funding}

No funding was received.

\section{Availability of data and materials}

The datasets used and/or analyzed during the current study are available from the corresponding author on reasonable request.

\section{Authors' contributions}

SC designed the experiments. SC and ZL performed the experiments. SL and BH analyzed data.SC drafted the manuscript and all authors reviewed and approved the manuscript.

\section{Ethics approval and consent to participate}

The study was approved by the Ethics Committee of the Jingzhou Central Hospital. All patients and their guardians provided written informed consent.

\section{Patient consent for publication}

Patients signed informed consent for publication.

\section{Competing interests}

The authors declare that they have no competing interests.

\section{References}

1. Botter SM, Neri D and Fuchs B: Recent advances in osteosarcoma. Curr Opin Pharmacol 16: 15-23, 2014.
2. Gattia M, Solari A, Pattarozzi A, Campanella C, Thellung S, Maniscalco L, De Maria R, Würth R, Corsaro A, Bajetto A, et al: In vitro and in vivo characterization of stem-like cells from canine osteosarcoma and assessment of drug sensitivity. Exp Cell Res 363: 48-64, 2018.

3. Bielack SS, Kempf-Bielack B, Delling G, Exner GU, Flege S, Helmke K, Kotz R, Salzer-Kuntschik M, Werner M, Winkelmann W, et al: Prognostic factors in high-grade osteosarcoma of the extremities or trunk: An analysis of 1,702 patients treated on neoadjuvant cooperative osteosarcoma study group protocols. J Clin Oncol 20: 776-790, 2002.

4. Luetke A, Meyers PA, Lewis I and Juergens H: Osteosarcoma treatment-where do we stand? A state of the art review. Cancer Treat Rev 40: 523-532, 2014.

5. Kansara M and Thomas DM: Molecular pathogenesis of osteosarcoma. DNA Cell Biol 26: 1-18, 2007.

6. Li JP, Liu LH, Li J, Chen Y, Jiang XW, Ouyang YR, Liu YQ, Zhong $\mathrm{H}, \mathrm{Li} \mathrm{H}$ and Xiao T: Microarray expression profile of long noncoding RNAs in human osteosarcoma. Biochem Biophys Res Commun 433: 200-206, 2013.

7. Gao JZ, Li J, DU JL and Li XL: Long non-coding RNA HOTAIR is a marker for hepatocellular carcinoma progression and tumor recurrence. Oncol Lett 11: 1791-1798, 2016.

8. Ning S, Zhang J, Wang P, Zhi H, Wang J, Liu Y, Gao Y, Guo M, Yue M, Wang L and Li X: Lnc2Cancer: A manually curated database of experimentally supported lncRNAs associated with various human cancers. Nucleic Acids Res 44: D980-D985, 2016.

9. Augoff K, McCue B, Plow EF and Sossey-Alaoui K: miR-31 and its host gene lncRNA LOC554202 are regulated by promoter hypermethylation in triple-negative breast cancer. Mol Cancer 11: 5, 2012.

10. Wang Y, Zhao W and Fu Q: miR-335 suppresses migration and invasion by targeting ROCK1 in osteosarcoma cells. Mol Cell Biochem 384: 105-111, 2013.

11. Livak KJ and Schmittgen TD: Analysis of relative gene expression data using real-time quantitative PCR and the 2(-Delta Delta C(T)) method. Methods 25: 402-408, 2001.

12. Harting MT, Blakely ML, Jaffe N, Cox CS Jr, Hayes-Jordan A, Benjamin RS, Raymond AK, Andrassy RJ and Lally KP: Long-term survival after aggressive resection of pulmonary metastases among children and adolescents with osteosarcoma. J Pediatr Surg 41: 194-199, 2006.

13. Hori SS and Gambhir SS: Mathematical model identifies blood biomarker-based early cancer detection strategies and limitations. Sci Transl Med 3: 109ra-116ra, 2011.

14. Grammatikakis I, Panda AC, Abdelmohsen K and Gorospe M: Long noncoding RNAs (lncRNAs) and the molecular hallmarks of aging. Aging (Albany NY) 6: 992-1009, 2014.

15. Mayfield RD: Emerging roles for ncRNAs in alcohol use disorders. Alcohol 60: 31-39, 2017

16. Wang J, Qiu M, Xu Y, Li M, Dong G, Mao Q, Yin R and Xu L: Long noncoding RNA CCAT2 correlates with smoking in esophageal squamous cell carcinoma. Tumour Biol 36: 5523-5528, 2015.

17. Rath $\mathrm{N}$ and Olson MF: Rho-associated kinases in tumorigenesis: Re-considering ROCK inhibition for cancer therapy. EMBO Rep 13: 900-908, 2012.

18. Zhou X, Wei M and Wang W: MicroRNA-340 suppresses osteosarcoma tumor growth and metastasis by directly targeting ROCK1. Biochem Biophys Res Commun 437: 653-658, 2013.

19. Liu X, Choy E, Hornicek FJ, Yang S, Yang C, Harmon D, Mankin $\mathrm{H}$ and Duan Z: ROCK1 as a potential therapeutic target in osteosarcoma. J Orthop Res 29: 1259-1266, 2011.

20. Li E, Zhang J, Yuan T and Ma B: MiR-145 inhibits osteosarcoma cells proliferation and invasion by targeting ROCK1. Tumour Biol 35: 7645-7650, 2014.

This work is licensed under a Creative Commons Attribution-NonCommercial-NoDerivatives 4.0 International (CC BY-NC-ND 4.0) License. 